\title{
Identification of Aircraft Noise During Acoustic Monitoring by Using 3D Sound Probes
}

\author{
M. KŁACZYŃSKI* \\ AGH University of Science and Technology, Faculty of Mechanical Engineering and Robotics, \\ Department of Mechanics and Vibroacoustics, Al. A. Mickiewicza 30, 30-059 Krakow, Poland
}

\begin{abstract}
Undertaking long-term acoustic measurements on sites located near an airport is related to a problem of large quantities of recorded data which very often represents information not related to flight operations. In such areas, usually defined as zones of limited use, other sources of noise often exist such as roads or railway lines treated in such context as an acoustic background. Manual verification of such recorded data is a costly and timeconsuming process. Automatic differentiation of the tested noise source from background and precise recognition of quantitative impact of aircraft noise on the acoustic climate in a particular area is an important task. This paper presents the idea of a method that can be used for identifying aircraft operations (flights, take-offs, landings) supported by experimental studies carried out with the use of 3D Microflown sound intensity probe and SoundField ST350 ambisonic microphone. The proposed method is based on determining the spatial sound intensity vector in the tested acoustic field during a monitoring timespan. On the grounds of this information, aircraft operations are marked in a continuous record of noise events.
\end{abstract}

DOI: 10.12693/APhysPolA.125.A-144

PACS: 43.50.Yw, 43.58.+z, 43.60.Jn

\section{Introduction}

Noise is one of major environmental problems in inhabited areas of the world. In Poland, development of domestic communication has become one of the main sources of noise hazards in the environment over the last 20 years. The road-transportation (traffic) noise has been the dominant factor in the change of acoustic climate due to its widespread nature and prolonged effect. However, noise generated by flight, takeoff, and landing of aircrafts in close proximity of airports has also been an important factor here. Since the Poland's accession to the European Union and the Polish sky becoming fully accessible for aircraft carriers, the air traffic has been growing rapidly. The growth of the air traffic in the coming years is predicted to be lower than current one, according to the forecast of the Civil Aviation Office [1], however it will be still higher than the European average. In 2012, Polish airports tripled in the number of passengers served (24.4 million), comparing to 2003 (7.1 million), which translates into approximately doubled overall number of air operations. Estimations for 2020 are that the number of passengers being served will increase to approximately 38 million, which will result in further doubling of air operations (comparing to the current level).

While exploiting the environment by emission of significant quantities of acoustic energy, according to European Union Directive 2002/49/EC the management of an airport (alike as this of a road, a railway, or a port) is required to perform continuous measurement of such

*e-mail: maciej.klaczynski@agh.edu.pl emissions. Such studies are designed to collect information about the prevailing acoustic climate and to produce conclusions, reports, and maps of the areas most threatened with the limits being exceeded. Carrying out continuous monitoring of a particular area involves problems of large quantities of the recorded data, often representing the information unrelated to the studied source. In such area, usually defined as the zone of limited use, there are typically also other sources of noise, such as roads and railway lines, then treated as a background sound. Manual verification of related data is time-consuming and costly. Identification and separation of background noise sources becomes a crucial task for proper determination of noise levels.

The aim of current research was to check concept of method for identifying aircraft operations (flights, takeoffs, landings) noise based on determining spatial sound intensity vector in the tested acoustic field during a monitoring timespan. On this basis, aircraft operations can be marked in a continuous record of noise events. Experimental measurements were performed by using two different sound transducer - 3D Microflown sound intensity probe and SoundField ST350 ambisonic microphone.

\section{Specificity of an aircraft noise}

Each aircraft flying above an observer emits a characteristic acoustic signal. This signal can constitute the basis for the recognition of the aircraft as well as its flight trajectory. As compared with environmental hazard caused by other sources, the specificity of air noise is as follows [2]:

- noise influences affects relatively large areas, 
- aircraft are characterised by high levels of noise emission, but they are usually at a large distance from objects for which such noise is harmful,

- propagation path of sound waves (coming from above) makes impossible the application of effective environment protections against noise, available e.g. in the case of traffic noise.

Propagation of air noise in the environment is most often determined by using calculation procedures (e.g. INM [3]). However, the basis for assessment of the noise arduousness around airports consists in the measurement results. Such measurements are simultaneously performed in a few or a dozen or so characteristic points by means of professional monitoring stations. The following parameters are most often recorded in individual measuring points:

- time of event,

- sound exposure level $L_{A E}$,

- maximal sound level $L_{\text {Amax }}$,

- equivalent sound level $L_{A e q}$,

- duration of event $t_{10}$,

- sound pressure level as a function of time,

- description of event (or possible disturbances),

During acoustics measurements, the monitoring system requires additional information that uniquely describes the recorded acoustic event. In the case of short-term acoustic climate monitoring, such information (acoustic event markers) can be obtained directly from the measuring team. However, during long-term studies or continuous monitoring it is not possible. In such cases, the data from airport radar system can be used [4]. This means that the noise level is correlated with the information derived from aircraft routes (altitude, direction, speed) recorded by airport radar system. When the sound level reaches a threshold value and the radar system detects an aircraft in the vicinity of the measuring point, the noise source is classified as an aircraft. For example, Figure 1 shows a sample of the sound pressure level (SPL) course recorded using continuous noise monitoring station in urban areas located close to an airport and a railway line. The first two acoustic events are related to the aircraft flights (departure from the airport - Boeing 738 and Airbus 320 ), followed by passage of train EN57. These acoustic events have been attributed to the source manually using information coming from the virtual air traffic radar AirNav RadarBox. The waveforms of the sound pressure level for this three acoustic events are very similar, so it is not possible to identify the source of noise by observing only the course of SPL.

Another way of identifying the aircraft noise during acoustic monitoring is using a four-microphone array the task of which is to determine the source position with

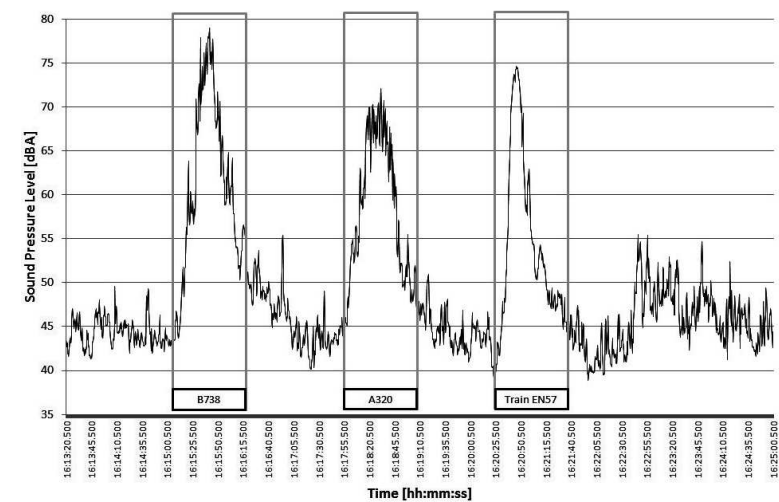

Fig. 1. The course of the sound pressure level (dBA) recorded by the continuous noise monitoring station located close by the airport.

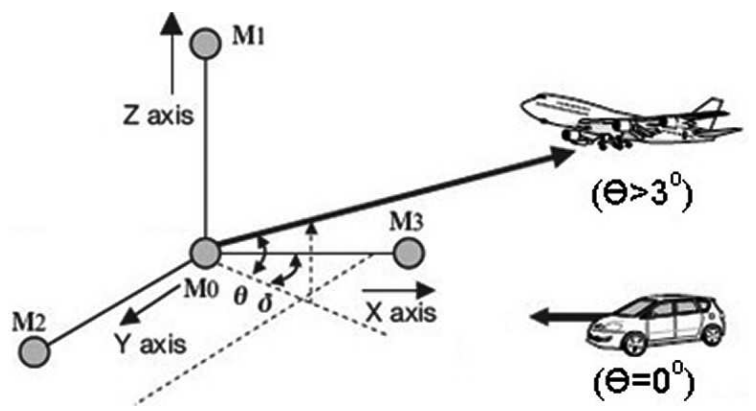

Fig. 2. Identification of aircraft noise by using four microphone array (after [5]).

respect to the receiver (angle of elevation and azimuth) and the fifth microphone necessary to conduct sound level measurements [5]. Figure 2 illustrates the main concept lying behind the proposed method of identification of aircraft noise sources by using such system.

Value of elevation angle $\theta$ is estimated according to the formula based on the sound wave arrival time delay in reaching two microphones - M0, M1:

$$
\theta=\arcsin \left(\frac{\tau c}{d}\right) .
$$

where: $\tau$ is the difference between the times of arrival of the sound at two microphones $\mathrm{M} 0$ and $\mathrm{M} 1, c$ is the sound velocity in air, and $d$ is the distance between microphones $\mathrm{M} 0$ and M1.

Azimuth angle $\phi$ is calculated using information from three microphones - M0, M2, and M3. When the elevation angle is greater than three degrees, the source is identified as an aircraft, and otherwise as a background sound.

One of possible alternative approaches to the issue, is a concept based on automatic "understanding" of sounds recorded by the measuring microphone (a part of the monitoring station) and referring to the advanced cognitive techniques based on the human auditory perception. In previous studies conducted by the authors $[2,6-10]$ on identification of noise sources and the type of airplane 
operations, automated pattern recognition methods were used. These were artificial neural networks (Multi-Layer Perceptron, Adaptive Resonance Theory, Self Organizing Maps), minimum distance classifiers (Nearest Neighbor, k-Nearest Neighbors and Nearest Mean), and a probabilistic method (statistical decision method based on a threshold of discrimination). But a prerequisite for (and also a disadvantage of) using these solutions is the necessity to collect a representative sample group of learners and perform a successful validation of the method.

\section{Spatial sound intensity vector}

The sound intensity $I$ is defined as the average value of the sound energy flux density level and is treated as a complex value (active and reactive intensity). It can also be described as a time-varying product of the sound pressure $p$ and the medium particle velocity $v$ [11]. Average value (over time $T$ ) of the sound intensity is given by

$$
I_{n}=\frac{1}{T} \int_{0}^{T} p_{n}(t) \boldsymbol{v}_{n}(t) \mathrm{d} t\left[\mathrm{~W} \mathrm{~m}{ }^{-2}\right],
$$

where $n=\{X, Y, Z\}$ is the orthogonal direction.

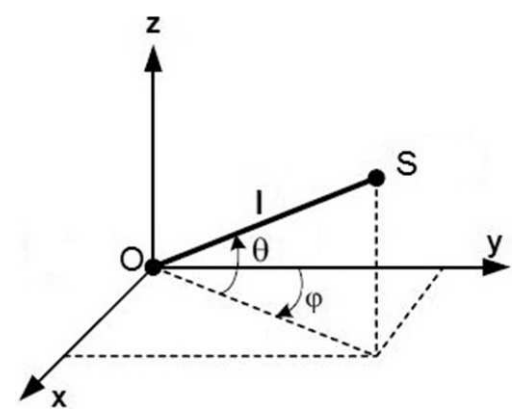

Fig. 3. Sound intensity vector in the spherical coordinate system.

Using geometrical relationships in the Cartesian coordinate system $X, Y, Z$ (three-dimensional Euclidean space), while assuming the acoustic wave propagation in free field between the sound source (object) $S$ and the observer $O$ (Fig. 3), the sound intensity can be represented as a scalar value according to the formula

$$
I=\sqrt{I_{x}^{2}+I_{y}^{2}+I_{z}^{2}},
$$

while the azimuth angle $\varphi$ can be calculated as

$$
\varphi=\operatorname{arctg}\left(\frac{I_{y}}{I_{x}}\right),
$$

and the elevation angle $\theta$ as

$$
\theta=\arcsin \left(\frac{I_{z}}{\sqrt{I_{x}^{2}+I_{y}^{2}}}\right) .
$$

The method proposed in this research is based on determining spatial sound intensity vector in the tested acoustic field during the acoustic monitoring. The timeaveraged sound intensity vector $I=\left[I_{x}, I_{y}, I_{z}\right]$ a and the azimuthal angle $\varphi$ and elevation angle $\theta$ are estimated. Two types of sound transducers were used, 3D Microflown USP sound intensity probe [12] and SoundField ST350 ambisonic microphone [13]. All calculations were done in post-processing mode, based on the recorded time waveform of the acoustic velocity and the sound pressure. Importantly, SoundField ST350 is not a sound intensity probe but a microarray of four microphones (capsules) arranged in vertices of a tetrahedron. Due to the location of the capsules and the signal processing method, ST350 is called the first-order ambisonic microphone. The resulting signal set is called B-format and contains the complete information on the sound field around the microphone during registration. Three signals of B-format correspond to microphones with bidirectional characteristics oriented along directions $X, Y$, and $Z$, and one signal $W$ corresponds to an omnidirectional microphone. Therefore, B-Format vector can represent components of the sound intensity vector Iaccording to the following formulae:

$$
\begin{aligned}
& p=\sqrt{2} W, \\
& v=\left[\begin{array}{c}
X \\
Y \\
Z
\end{array}\right] .
\end{aligned}
$$

One of the first studies related to location of a sound source using the SoundField microphone under laboratory conditions was carried out and presented in [14]. The first tests associated with detection and location of an aircraft as the sound source using the Microflown probe were conducted by its inventors and presented in $[15-17]$.

\section{Experimental study}

Experimental studies of automatic classification of aircraft noise during long-term acoustic monitoring using spatial sound probes were conducted in village Rząska near Kraków, Poland. The measuring point was in the close vicinity of a railway line and route of departures and arrivals to "Kraków Airport". The multi-channel signal acquisition LMS SCADAS MOBILE system with 8channel data acquisition LMS VM8-E module was used for data recording. The system ensures recording waveforms of sound pressure and particle velocity with dynamics exceeding $140 \mathrm{~dB}$ and $51,200 \mathrm{~Hz}$ sampling frequency. All further calculations necessary to determine the parameters described above were carried out in MATLAB 7.12. Parallel measurements were performed using a mobile noise monitoring station equipped with a sound meter and SVAN 958 analyser with GRAS 40AE microphone in an all-weather cover. Short-term example of the waveform sound pressure level is shown in Figure 1. Measurements were taken during the day, at outdoor temperature ranging from a maximum of $20^{\circ} \mathrm{C}$ to a minimum of $16^{\circ} \mathrm{C}$, atmospheric pressure $1020 \mathrm{hPa}$, and wind speed not exceeding $1 \mathrm{~m} / \mathrm{s}$. Both SoundField microphone and 
Microflown probe were equipped with windscreens provided by the manufacturers.

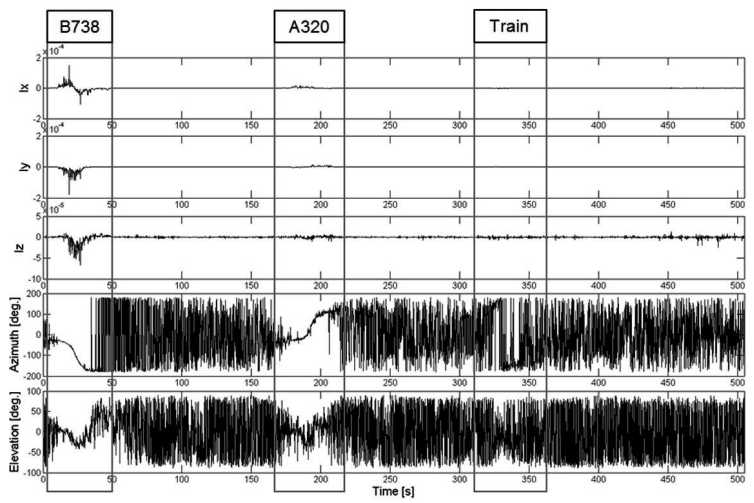

Fig. 4. The course of sound intensity $I x, I y, I z$, azimuth and elevation angle of $I$ vector averaged over time $(0.125 \mathrm{~s})$ Measurements taken with Microflown probe.

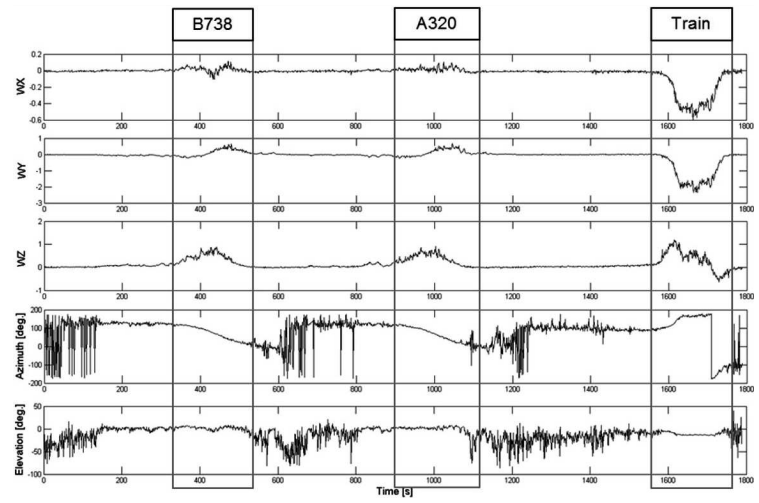

Fig. 5. The course of B-format vector components, azimuth, and elevation angle of B-format vector averaged over time $(0.125 \mathrm{~s})$ Measurements taken by SoundField ST350 microphone.

Figure 4 shows an example of averaged waveforms of sound intensity (with time averaging of $0.125 \mathrm{~s}$ ) registered by Microflown probe, each for the $x, y, z$ direction, as well as the courses of azimuth and elevation angle of the sound intensity vector in the adopted coordinate system (pointing from the receiver to the sound source).

In Figure 4, vertical lines indicate time intervals of occurrence of noise events associated with aircraft flights (departure from the airport, Boeing 738 and Airbus 320) and passage of a passenger train. In the case of plane flights, change of the azimuth angle ranges from $0^{\circ}$ to $180^{\circ}$ (flight along the horizon), while the elevation angle increases from $0^{\circ}$ to about $50^{\circ}$ and then decreases to $0^{\circ}$ (takeoff, flight over the measurement point, and flight to the horizon). In the case of the train passing near the measuring point, the azimuth angle starts from about $180^{\circ}$ and then turns to $-180^{\circ}$ (arrival, passing near the point, and departure), and the elevation angle is negative (the railway was in a ravine, below the level on which the measurement point was located).

Figure 5 shows an example of averaged waveforms of B-format vector components representing the sound intensity vector $\boldsymbol{I}$ components (registered by Soundfiled ST350 microphone with time averaging of $0.125 \mathrm{~s}$ ), each for the $x, y, z$ direction, as well as the courses of azimuth and elevation angle of the sound intensity vector in the adopted coordinate system (pointing from the receiver to the sound source).

In Figure 5, vertical lines indicate time intervals of occurrence of noise events associated with aircraft flights (arrival at the airport, Boeing 737 and Boeing 738), and passage of a passenger train. In the case of plane flights, change of the azimuth angle ranges from $150^{\circ}$ to $0^{\circ}$ (passing along the horizon), with the angle of elevation increasing from negative (around $-50^{\circ}$ ) to positive values (about $5^{\circ}$ ). In the case of a train passing near the measuring point, at first the azimuth angle was about $180^{\circ}$ and then turned to $-180^{\circ}$ (arrival, passing near the measurement point, and departure), and the elevation angle was negative (the railway was in a ravine, below the level on which the measurement point was located).

\section{Conclusions}

This paper presents preliminary results of new experimental research project related to the use of sound source localization methods in automatic classification of aircraft noise during acoustic environmental monitoring. 3D Microflown sound intensity probe and SoundField ST350 ambisonic microphone were used. Results of the algorithm used to determine the position angle of sound sources in the adopted coordinate system are quite promising. The elevation angle of an aircraft near the airport area can be estimated with good accuracy by using the Microflown probe. For measurements carried out with the use of SoundField ST350 microphone, estimation of the elevation angle is less accurate in comparison with actual position of the aircraft. Further work in this area will focus on developing optimal algorithm for processing the recorded signals in order to make the best possible estimate of the sound source spatial parameters which are related to aircraft flight operations.

\section{Acknowledgments}

This paper has been written and the respective research work undertaken within the project 2011/01/D/ST6/07178 (National Science Centre).

\section{References}

[1] Civil Aviation Office, http://www.ulc.gov.pl/, 01.07.2013.

[2] W. Wszołek, M. Kłaczyński, Mech. Contr. 29, 4, (2010). 
[3] He, Dinges, Hemann, Rickel, Mirsky, Roof, Boeker, Gerbi, Senzing, Integrated Noise Model (INM) Version 7.0 user's guide, Office of Environment and Energy, Washington 2007.

[4] Airport environment management, Bruel\&Kjaer, Denmark 2013.

[5] Environmental Sound Monitoring for Aircraft Noise Measurement, Rion 2013.

[6] M. Kłaczyński, T. Wszołek, Acta Phys. Pol. A 121, A-179 (2012).

[7] M. Kłaczyński, T. Wszołek, W. Batko, in: 59th Open Seminar of Acoustics, Poznań-Boszkowo 2012, p. 113.

[8] M. Kłaczyński, T. Wszołek, W. Batko, in: 9th European Conference on Noise Control EURONOISE, Prague 2012, p. 549.

[9] M. Kłaczyński, T. Wszołek, W. Batko, in: 58th Open Seminar of Acoustics, Gdańsk-Jurata 2011, p. 347.

[10] W. Wszołek, M. Kłaczyński, T. Wszołek, in: 39th International Congress and Exposition on Noise Control Engineering INTERNOISE, Lisbon 2010.
[11] S. Weyna, Acoustic energy distribution of real sources, WNT, Warszawa 2005, p. 99, (in Polish).

[12] Datasheet and User Manual for Microflown USP probe regular.

[13] SoundField ST350 Portable Microphone System, user guide ver. 1.03.

[14] J. Wierzbicki, P. Małecki, J. Wiciak, Acta Phys. Pol. A 123, 1114 (2013).

[15] H-E. de Bree, J. Wind, P. de Theije, in: 40th International Congress and Exposition on Noise Control Engineering INTERNOISE, Osaka, 2011.

[16] H-E. de Bree, J. Wind, E. Tijs, in: 39th International Congress and Exposition on Noise Control Engineering INTERNOISE, Lisbon 2010.

[17] H-E. de Bree, J. Wind, S. Sadasivan, Broad banded acoustic vector sensors for passive monitoring of aircraft, DAGA, 2009. 